\title{
Clinical profile and factors associated with mortality in hospitalized patients with HIV/AIDS: a retrospective analysis from Tripoli Medical Centre, Libya, 2013
}

N.S. Shalaka, ${ }^{1,2}$ N.A Garred, ${ }^{1}$ H.T. Zeglam, ${ }^{1}$ S.A. Awasi, ${ }^{1}$ L.A. Abukathir, ${ }^{1}$ M.E Altagdi ${ }^{7}$ and A.A. Rayes ${ }^{1,2}$

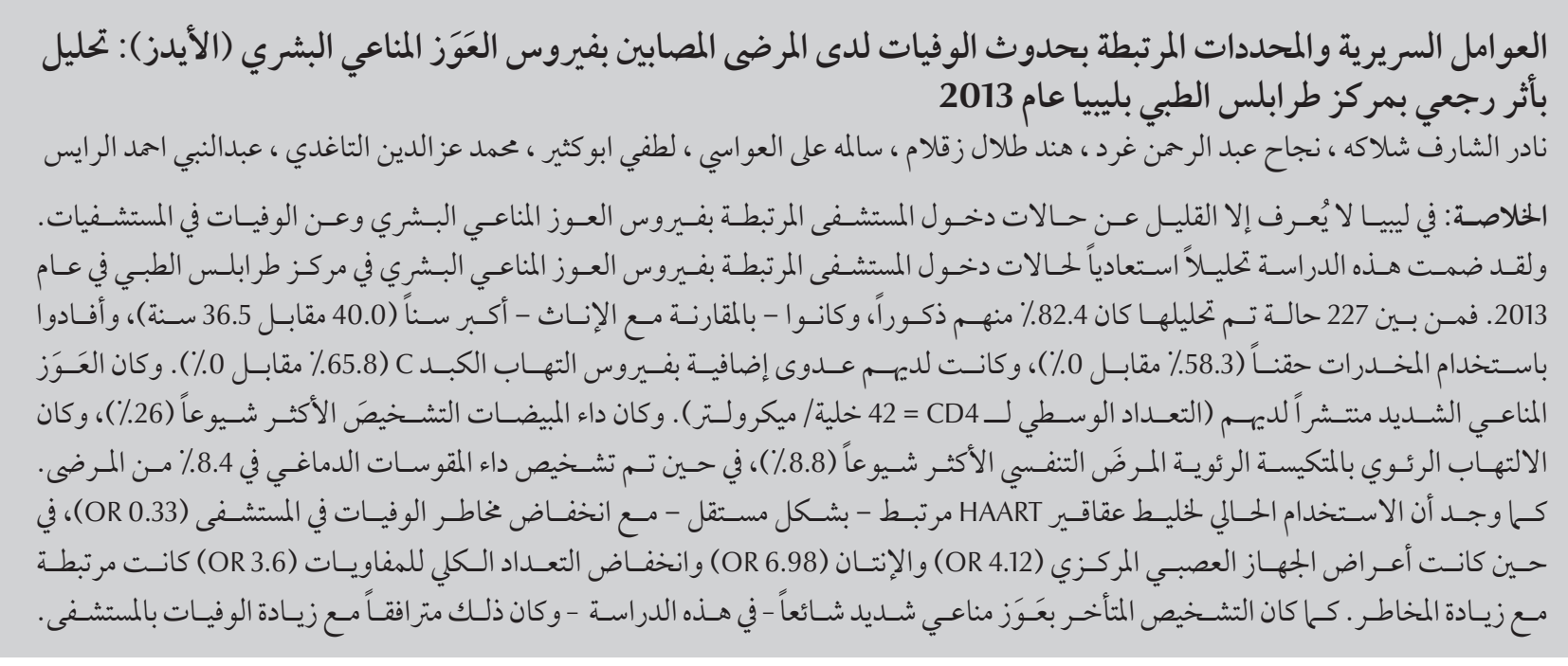

ABSTRACT In Libya, little is known about HIV-related hospitalizations and in-hospital mortality. This was a retrospective analysis of HIV-related hospitalizations at Tripoli Medical Centre in 2013. Of 227 cases analysed, 82.4\% were males who were significantly older (40.0 versus 36.5 years), reported injection drug use $(58.3 \%$ versus $0 \%$ ) and were hepatitis $C$ virus co-infected (65.8\% versus $0 \%$ ) compared with females. Severe immunosuppression was prevalent (median CD4 count $=42 \mathrm{cell} / \mu \mathrm{L})$. Candidiasis was the most common diagnosis (26.0\%); Pneumocystis pneumonia was the most common respiratory disease $(8.8 \%)$, while cerebral toxoplasmosis was diagnosed in $8.4 \%$ of patients. Current HAART use was independently associated with low risk of in-hospital mortality (OR 0.33), while central nervous system symptoms (OR 4.12), sepsis (OR 6.98) and low total lymphocyte counts (OR 3.60) were associated with increased risk. In this study, late presentation with severe immunosuppression was common, and was associated with significant in-hospital mortality.

Profil clinique et facteurs associés à la mortalité chez des patients hospitalisés vivant avec le VIH/sida : analyse rétrospective du Centre médical de Tripoli (Libye), 2013

RÉSUMÉ En Libye, les connaissances sur les hospitalisations et la mortalité en milieu hospitalier liées au VIH sont rares. Nous avons procédé à une analyse rétrospective des hospitalisations liées au VIH au centre médical de Tripoli en 2013. Sur 227 cas analysés, 82,4 \% étaient des hommes nettement plus âgés (40,0 contre 36,5 ans), qui déclaraient s'injecter des drogues (58,3\% contre $0 \%)$ et qui étaient atteints d'une co-infection par le virus de l'hépatite C (65,8 \% contre $0 \%$ )comparativementauxfemmes.L'immunosuppressionsévèreétaitprévalente(numérationdeslymphocytes T-CD4 = 42 cellules/ $\mu \mathrm{L})$. Le diagnostic le plus fréquent était la candidose $(26,0 \%)$; la pneumonie à Pneumocystis était la maladie respiratoire la plus fréquente $(8,8 \%)$, tandis que la toxoplasmose cérébrale était diagnostiquée chez $8,4 \%$ des patients). Un traitement antirétroviral hautement actif en cours était indépendamment associé à un faible risque de mortalité en milieu hospitalier (OR 0,33), tandis que les symptômes du système nerveux central (OR $4,12)$, la septicémie $(O R 6,98)$ et les faibles numérations lymphocytaires totales $(O R 3,60)$ étaient associés à un risque accru. Dans cette étude, une présentation tardive accompagnée d'une immunosuppression sévère était fréquente, et était associée à une mortalité élevée en milieu hospitalier. 


\section{Introduction}

Human immunodeficiency virus (HIV) and its associated acquired immune deficiency syndrome (AIDS) continue to exact a significant deal of morbidity and mortality worldwide, with over 35 million people infected by the year 2013 (1). In developed countries, the wide availability of highly active antiretroviral therapy (HAART) since the mid-1990s has significantly reduced AIDS-related hospitalizations and mortality, and improved the overall survival among affected individuals (2-5). It has also reduced rates of AIDS-defining caused by opportunistic infections, and changed the spectrum of HIV-related hospitalizations to become mostly due to chronic and non-AIDS-defining conditions, such as cardiovascular diseases and malignancy (6-8).

By contrast, AIDS is still the main reason for hospitalization in developing countries, with various opportunistic infections playing major roles in HIVrelated morbidity and mortality (9-11). With such variations in the spectrum of HIV-related diseases, findings from developed countries might not be generalizable to our settings. In addition, although several studies have been conducted in other developing countries of Africa and Asia (12-17), the spectrum of diseases related to HIV/AIDS may have regional variations influenced by socioeconomic status, endemic infections, nutrition and availability of HAART and hepatitis coinfections. Knowledge regarding the spectrum of AIDS-defining illnesses and local prevalence of various opportunistic infections might be important for policy-makers and stakeholders to better manage limited screening, diagnostic and therapeutic resources and to plan actions to reduce morbidity and mortality. It might also be important in raising the awareness of physicians to most prevalent diagnoses and in taking decisions on empirical treatment and management plans.
With the paucity of published data on HIV/AIDS in Libya (18), the spectrum of AIDS-defining illnesses remains largely undetermined and little is known about the causes of HIV-related hospitalizations, clinical profiles and factors associated with mortality in hospitalized patients with HIV. In this study, we aimed to: define the spectrum of HIV-related causes of hospitalization; assess the profile of hospitalized HIV-positive adults; and examine the clinical and laboratory factors associated with in-hospital mortality among adults with HIV/AIDS admitted to Tripoli Medical Centre during the year 2013.

\section{Methods}

\section{Study site}

Tripoli Medical Centre is a university and tertiary referral hospital with a capacity of 1200 beds. It provides specialist medical services to patients from Tripoli and other cities in the western and central regions of Libya. The department of infectious diseases provides specialist HIV management as an ambulatory care as well as a 14-bed capacity dedicated for management of inpatients with HIV/AIDS.

\section{Study design}

This was a retrospective, observational analysis of HIV-related hospitalizations at the Centre from January to December 2013. As a routine screening, all admitted patients had their HIV, HBV and HCV status determined by 4thgeneration enzyme-linked immunosorbent assay testing. Patients who were 15 years of age or more at the time of hospitalization and with positive HIV test results were identified through the department's inpatient registry. Their case notes and discharge summaries were then reviewed and relevant data abstracted using a standard data collection form. Ethical approval was granted by the department of medicine and the scientific committee at Tripoli Medical Centre.

\section{Data collection}

The following variables were collected anonymously: demographics (age, sex, marital status) and HIV risk factors (HAART use; clinical signs and symptoms at the time of hospitalization); serological testing for hepatitis $\mathrm{C}(\mathrm{HCV})$ and hepatitis $\mathrm{B}$ virus (HBV) infections; haematological and biochemical laboratory results at the time of hospital admission and before rehydration, blood transfusion or administration of antibiotics; diagnosis during hospitalization; and status upon discharge (alive or dead).

\section{In-hospital diagnosis}

Although efforts were made to ascertain the diagnoses, the lack of some diagnostic facilities at the Centre and the presence of patients with advanced illness at the time of presentation might have prohibited invasive diagnostic procedures. In such instances, the following criteria were used to establish a diagnosis:

- pulmonary tuberculosis (TB): a positive sputum smear or culture for acid-fast bacilli or a compatible clinical presentation with suggestive findings on chest X-ray or computerized tomography scan and a response to anti-TB treatment.

- extrapulmonary TB: a histopathological diagnosis from an extrapulmonary site (e.g. lymph node; pleura) or a compatible clinical presentation with suggestive findings on imaging scans and a response to anti- $\mathrm{TB}$ treatment.

- cerebral toxoplasmosis: a compatible clinical presentation with suggestive findings on brain magnetic resonance imaging and a response to anti-toxoplasmosis treatment.

- progressive multifocal leucoencephalopathy: a compatible clinical presentation with suggestive findings on brain magnetic resonance imaging. 
- Pneumocystis jiroveci: a compatible clinical presentation with suggestive findings on chest X-ray/computerized tomography and a response to treatment (provided that TB was excluded).

- cryptococcal meningitis: a compatible clinical presentation with the detection of cryptococcal antigen in cerebrospinal fluid or serum.

- candidiasis: a compatible clinical presentation and/or findings on gastroscopy.

- sepsis: systemic inflammatory response syndrome with an identified focus of infection. Systemic inflammatory response syndrome was defined as 2 or more of the following: fever $>38^{\circ} \mathrm{C}$ or $<36{ }^{\circ} \mathrm{C}$; heart rate $>$ 90 beats per minute; respiratory rate $>20$ breaths per minute or an arterial carbon dioxide tension $<32 \mathrm{mmHg}$; and (d) white cell count $>12000$ or $<4000$ cells/ $\mu \mathrm{L}$.

All individuals with central nervous system symptoms or signs were screened for cryptococcal infection using a cryptococcal antigen test. Patients with retinitis had cytomegalovirus DNA test (polymerase chain reaction assay), whereas for patients with oesophagitis, gastritis, enteritis or colitis and not responding to medications, endoscopy, biopsy and histopathological examination for cytomegalovirus were performed. Severe immunosuppression was defined as CD4 count $<200$ cells/ $\mu \mathrm{L}$, and anaemia as haemoglobin $<13$ $\mathrm{mg} / \mathrm{dL}$ for males and $<12 \mathrm{mg} / \mathrm{dL}$ for females.

\section{Statistical analysis}

$R$, version 3.1.1, a language and environment for statistical computing ( $\mathrm{R}$ Foundation for Statistical Computing, Austria,) was used to conduct planned analyses.

Quantitative variables were summarized using the median and interquartile range (IQR), and bivariate analysis was done using Mann-WhitneyU-test. Categorical variables (grouped quantitative and qualitative variables) were summarized using frequency tables and percentages of total, while the bivariate analysis was performed using chisquared or Fisher exact tests; they were presented graphically using bar charts.

A stepwise logistic regression analyses was conducted to calculate adjusted odds ratios (OR). To maintain the assumption of independent observations in patients with two hospitalizations, only data available from the last admission were used.

Missing values were excluded during the analysis of continuous variables, but were included as a "missing" category during the grouped analysis. All analyses were 2-sided, and $P$-values of $<0.05$ were considered statistically significant.

\section{Results}

Our inpatient registry reported 340 hospitalizations in the year 2013; 227 were for individuals with positive HIV tests. During the study period, 211 HIV-positive patients were hospitalized; 16 of them had a re-hospitalization during the same year. The reason for re-hospitalization was different for all 16 patients (e.g. Pneumocystis jiroveci then skin Kaposi sarcoma 3 months after starting HAART).

\section{Demographic characteristics}

Table 1 summarizes the main demographic characteristics of the study group. The majority of patients were male $(187,82.4 \%)$ with a median age of 40 years (IQR 37.5-45 years) for males and 36.5 years (IQR 30-45 years $)$ for females $(P=0.01)$. Most male patients were single (55.6\%), reported injection drug use as a route of HIV transmission (58.3\%) and tested positive on HCV serology test (65.8\%). In contrast, most female patients were married (57.5\%) or widowed (22.5\%) and identified marital heterosexual contact as a route of HIV transmission (87.5\%), while none of them tested positive on HCV or HBV serological tests.

\section{Clinical symptoms and signs at admission}

The clinical symptoms and signs at time of admission are summarized in Table 2. Fever was the most common presenting symptom (42.3\%) followed by oral thrush $(29.1 \%)$. When analysed as groups of symptoms at time of admission, constitutional symptoms were the most common (59.9\%), followed by gastrointestinal (48.5\%) symptoms.

Although the majority of patients were known to have HIV infection before admission (85.9\%), most hospitalized patients were not on HAART (141, 62.0\%), of whom 103 (45.4\%) had never received HAART before.

\section{In-hospital diagnoses}

The spectrum of diagnoses is presented in Figure 1. Oral and oesophageal candidiasis was the most common diagnosis (26.0\%) followed by extrapulmonary TB (9.3\%) and sepsis (9.3\%). Pneumocystis jiroveci was the most common respiratory disease (8.8\%) whilst cerebral toxoplasmosis was the most commonly identified intracranial mass lesion (8.4\%).

\section{Laboratory test results at admission}

Tables 3 and 4 summarizethelaboratory test results at time of admission. Data on CD 4 counts were available for 169 inpatients $(74.4 \%)$. The median count was 42 cells/ $\mu \mathrm{L}$, with 150 patients ( $88.8 \%$ of available data) having CD4 counts $<200$ cells/ $\mu \mathrm{L}$. Anaemia was reported in 200 (88.0\%) patients and was severe in 39 (17.2\%), while thrombocytopenia was reported in 95 (41.9\%) patients. Both anaemia and thrombocytopenia were more commonly reported among males than females $(P=0.043$ and $P<$ 0.001 respectively). 


\begin{tabular}{|c|c|c|c|c|c|c|c|}
\hline \multirow{2}{*}{$\begin{array}{l}\text { Variable } \\
\text { Total admissions (No., \%) }\end{array}$} & \multicolumn{2}{|c|}{ Total } & \multicolumn{2}{|c|}{ Male } & \multicolumn{2}{|c|}{ Female } & \multirow[t]{2}{*}{$P$-value } \\
\hline & 227 & 100.0 & 187 & 82.4 & 40 & 17.6 & \\
\hline Age (years) [Median, (IQR)] & 40 & $(36-45)$ & 40 & $(37.5-45)$ & 36.5 & $(30-45)$ & $0.01^{\mathrm{a}}$ \\
\hline Marital status (No., \%) & & & & & & & $<0.001^{\mathrm{c}}$ \\
\hline Single & 110 & 48.5 & 104 & 55.6 & 6 & 15.0 & \\
\hline Married & 84 & 37.0 & 61 & 32.6 & 23 & 57.5 & \\
\hline Divorced & 10 & 4.4 & 9 & 4.8 & 1 & 2.5 & \\
\hline Widowed & 9 & 4.0 & 0 & 0.0 & 9 & 22.5 & \\
\hline Unknown & 14 & 6.2 & 13 & 7.0 & 1 & 2.5 & \\
\hline New HIV diagnosis (No., \%) & 32 & 14.1 & 23 & 12.3 & 9 & 22.5 & $0.15^{\mathrm{b}}$ \\
\hline HIV risk factors (No., \%) & & & & & & & $<0.001^{\mathrm{c}}$ \\
\hline Injection drug use & 109 & 48.0 & 109 & 58.3 & 0 & 0.0 & \\
\hline Heterosexual & 40 & 17.6 & 5 & 2.7 & 35 & 87.5 & \\
\hline Homosexual & 2 & 0.9 & 2 & 1.1 & 0 & 0.0 & \\
\hline Unknown & 76 & 33.5 & 71 & 38 & 5 & 12.5 & \\
\hline HAART use (No., \%) & & & & & & & $0.8^{c}$ \\
\hline Never & 103 & 45.4 & 83 & 44.4 & 20 & 50.0 & \\
\hline Current & 82 & 36.1 & 69 & 36.9 & 13 & 32.5 & \\
\hline Defaulted & 38 & 16.7 & 32 & 17.1 & 6 & 15.0 & \\
\hline Unknown & 4 & 1.8 & 3 & 1.6 & 1 & 2.5 & \\
\hline $\begin{array}{l}\text { Duration of hospital stay (days) } \\
\text { [Median, (IQR)] }\end{array}$ & 8 & $(3-16)$ & 9 & $(3-16)$ & 7 & $(3-16.2)$ & $0.61^{\mathrm{a}}$ \\
\hline HCV-Ab positive (No., \%) & 123 & 54.2 & 123 & 65.8 & 0 & 0.0 & $<0.001^{\mathrm{b}}$ \\
\hline HbsAg positive (No., \%) & 11 & 4.8 & 11 & 5.9 & 0 & 0.0 & $0.22^{\mathrm{c}}$ \\
\hline Hospital mortality (No., \%) & 85 & 37.4 & 76 & 40.6 & 9 & 22.5 & $0.049^{b}$ \\
\hline $\begin{array}{l}\text { Death within } 48 \text { h of admission } \\
\text { (No., \%) }\end{array}$ & 30 & 13.2 & 28 & 15.0 & 2 & 5.0 & $0.15^{b}$ \\
\hline
\end{tabular}

${ }^{a}$ Mann-Whitney $U$ test; ${ }^{b}$ Chi-squared-test; ${ }^{c}$ Fisher exact test.

$I Q R=$ interquartile range; $H A A R T=$ highly active antiretroviral therapy; $H C V$-Ab =anti-hepatitis C virus antibody; $H B V$ s $A g=$ hepatitis $B$ virus surface antigen .

\section{In-hospital mortality}

The median duration of hospital stay was 8 days and overall mortality was high ( 85 patients, $37.4 \%)$, with a significantly higher rate among males than females (40.6\% versus $22.5 \%$; $P=$ 0.049) (Table 1). Just over one-third of patients (30,35\% of all deaths) died within 48 hours of hospital admission.

\section{Factors associated with in- hospital mortality}

A total of 184 (87.2\%) patients with complete data were included in a stepwise logistic regression analysis, and results on independent risk factors are summarized in Table 5. Current HAART use was independently associated with a low risk of in-hospital mortality (OR 0.33; 95\% CI: 0.14-0.8; $P=0.015)$. Central nervous system symptoms (OR 4.12; 95\% CI: 1.77-9.6; $P=0.001)$, sepsis (OR 6.98; 95\% CI = 1.71-28.44; $P=0.007)$, hyponatraemia (OR 2.69; 95\% CI: 1.12-6.44; $P=$ 0.023 ) and low total lymphocyte counts (OR 3.6; 95\% CI: $1.36-9.52 ; P=0.01$ ) were associated with increased risk.

\section{Discussion}

In 2013 at Tripoli Medical Centre, AIDS-defining opportunistic infections were the most common reasons for HIV-related hospitalizations. Inpatients were mostly middle-aged men who were known to be HIV-positive before admission; a finding similar to previous reports from both developed and developing countries $(7,9)$. HIV-related hospitalizations were mostly among patients in their economically productive years, therefore increasing the socioeconomic burdens on the patients and their families.

Injection drug use was the most commonly identified HIV risk factor among males, with a concomitantly high prevalence of HCV coinfection. Although this is different to findings from developed (7) and other developing (19) countries where homosexual and heterosexual transmissions were the most common respectively, it was consistent with a previous report on the high prevalence of HIV/HCV coinfection among injection drug users in Libya 


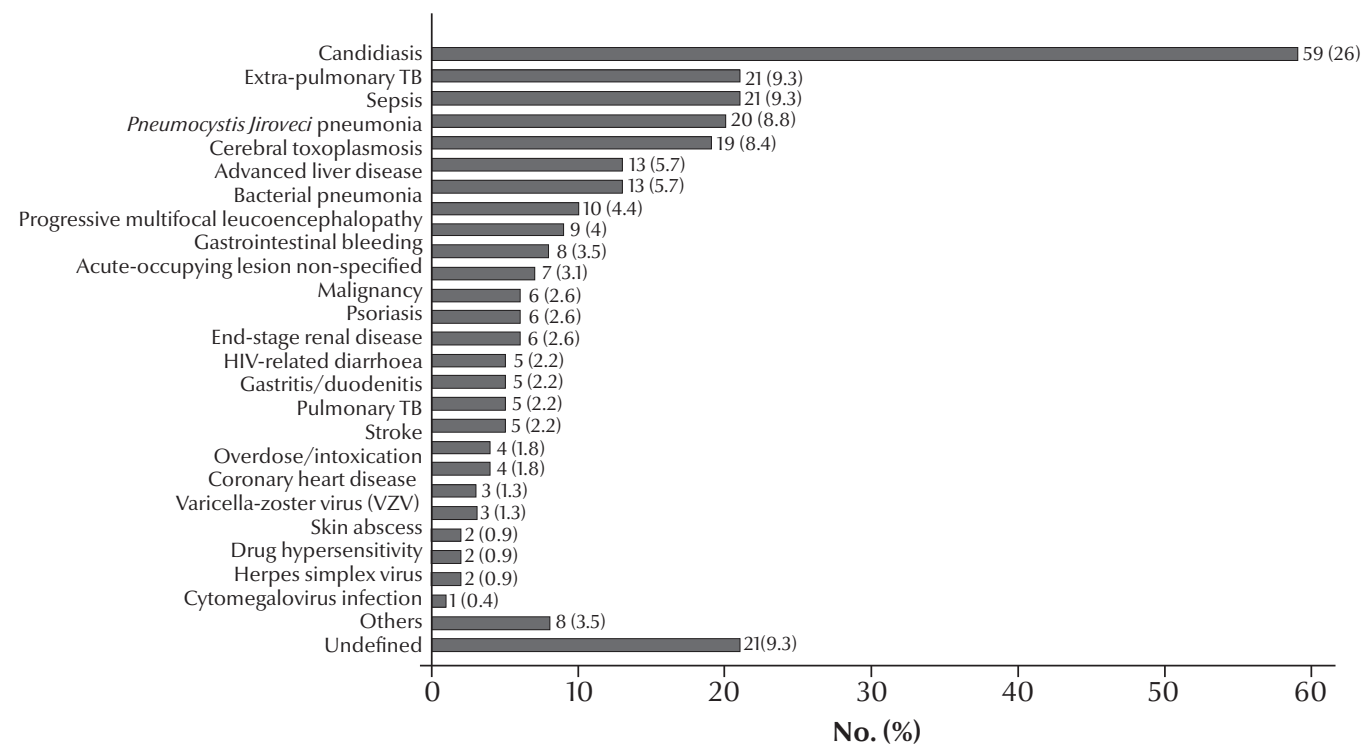

Figure 1 Spectrum of in-hospital diagnoses of the studied HIV/AIDS patients

\begin{tabular}{|c|c|c|c|c|c|c|c|}
\hline \multirow[t]{2}{*}{ Clinical presentation } & \multicolumn{2}{|c|}{$\begin{array}{c}\text { Total } \\
(n=227)\end{array}$} & \multicolumn{2}{|c|}{$\begin{array}{c}\text { Males } \\
(n=187)\end{array}$} & \multicolumn{2}{|c|}{$\begin{array}{c}\text { Females } \\
(n=40)\end{array}$} & \multirow[t]{2}{*}{$P$-value } \\
\hline & No. & $\%$ & No. & $\%$ & No. & $\%$ & \\
\hline Fever & 96 & 42.3 & 82 & 43.9 & 14 & 35.0 & $0.39^{\mathrm{a}}$ \\
\hline Weight loss & 35 & 15.4 & 34 & 18.2 & 1 & 2.5 & $0.024^{\mathrm{a}}$ \\
\hline Night sweating & 17 & 7.5 & 17 & 9.1 & 0 & 0.0 & $0.048^{\mathrm{b}}$ \\
\hline Lymphadenopathy & 12 & 5.3 & 11 & 5.9 & 1 & 2.5 & $0.7^{b}$ \\
\hline Oral thrush & 66 & 29.1 & 54 & 28.9 & 12 & 30.0 & $1^{\mathrm{a}}$ \\
\hline Skin lesions & 22 & 9.7 & 18 & 9.6 & 4 & 10.0 & $1^{b}$ \\
\hline Cough & 65 & 28.6 & 52 & 27.8 & 13 & 32.5 & $0.69^{a}$ \\
\hline Dyspnoea & 65 & 28.6 & 52 & 27.8 & 13 & 32.5 & $0.69^{a}$ \\
\hline Pleural effusion & 10 & 4.4 & 10 & 5.3 & 0 & 0.0 & $0.22^{\mathrm{b}}$ \\
\hline Decreased level of consciousness & 61 & 26.9 & 55 & 29.4 & 6 & 15.0 & $0.095^{\mathrm{a}}$ \\
\hline Focal neurological deficit & 21 & 9.3 & 12 & 6.4 & 9 & 22.5 & $0.004^{\mathrm{b}}$ \\
\hline Convulsions & 17 & 7.5 & 15 & 8.0 & 2 & 5.0 & $0.74^{\mathrm{b}}$ \\
\hline Vomiting & 38 & 16.7 & 32 & 17.1 & 6 & 15.0 & $0.93^{\mathrm{a}}$ \\
\hline Diarrhoea & 39 & 17.2 & 34 & 18.2 & 5 & 12.5 & $0.53^{\mathrm{a}}$ \\
\hline Abdominal pain & 18 & 7.9 & 11 & 5.9 & 7 & 17.5 & $0.022^{\mathrm{b}}$ \\
\hline Jaundice & 15 & 6.6 & 14 & 7.5 & 1 & 2.5 & $0.48^{\mathrm{b}}$ \\
\hline Hepatomegaly & 16 & 7.0 & 15 & 8.0 & 1 & 2.5 & $0.32^{\mathrm{b}}$ \\
\hline Splenomegaly & 6 & 2.6 & 6 & 3.2 & 0 & 0.0 & $0.59^{b}$ \\
\hline Ascites & 17 & 7.5 & 17 & 9.1 & 0 & 0.0 & $0.048^{\mathrm{b}}$ \\
\hline Dysphagia & 30 & 13.2 & 24 & 12.8 & 6 & 15.0 & 0.91 \\
\hline Haematemesis & 10 & 4.4 & 10 & 5.3 & 0 & 0.0 & $0.22^{\mathrm{b}}$ \\
\hline Melaena & 9 & 4.0 & 9 & 4.8 & 0 & 0.0 & $0.37^{b}$ \\
\hline
\end{tabular}

${ }^{a}$ Chi-squared-test; ${ }^{b}$ Fisher exact test. 


\begin{tabular}{|c|c|c|c|c|}
\hline Variable & $\begin{array}{c}\text { Total } \\
\text { Median (IQR) }\end{array}$ & $\begin{array}{c}\text { Males } \\
\text { Median (IQR) }\end{array}$ & $\begin{array}{c}\text { Females } \\
\text { Median (IQR) }\end{array}$ & $P$-value ${ }^{a}$ \\
\hline CD4 count $($ cells $/ \mu \mathrm{L})(n=169)$ & $42(8-87)$ & $41(8-84)$ & $43(6-151)$ & 0.99 \\
\hline WC count $\left(\right.$ cells $\left.\times 10^{3} / \mu \mathrm{L}\right)(n=218)$ & $5.1(3.2-7.9)$ & $5.1(3.2-7.7)$ & $5.2(3.8-8.4)$ & 0.53 \\
\hline $\mathrm{Hb}(\mathrm{g} / \mathrm{dL})(n=218)$ & $10.1(8.6-12.0)$ & $10.3(8.5-12.0)$ & $10.0(9.0-11.4)$ & 0.9 \\
\hline Platelet count $\left(\times 10^{3} / \mu \mathrm{L}\right)(n=218)$ & $166(90-237)$ & $154(83-225)$ & $227(164-301)$ & $<0.001$ \\
\hline $\mathrm{BUN}(\mathrm{mg} / \mathrm{dL})(n=202)$ & $15.5(10-27.5)$ & $17.0(11-29)$ & $10.5(9-16)$ & 0.003 \\
\hline Creatinine $(\mathrm{mg} / \mathrm{dL})(n=208)$ & $0.8(0.6-1.1)$ & $0.9(0.7-1.1)$ & $0.6(0.5-0.8)$ & $<0.001$ \\
\hline Sodium $(\mathrm{mmol} / \mathrm{L})(n=209)$ & $133(129-136)$ & $132(128-136)$ & 136 (134-138) & $<0.001$ \\
\hline Potassium (mmol/L) $(n=209)$ & $4.0(3.6-4.5)$ & $4.0(3.7-4.6)$ & $4.0(3.5-4.2)$ & 0.11 \\
\hline $\operatorname{ALT}(\mathrm{U} / \mathrm{L})(n=152)$ & $39.5(29-54)$ & $41.0(29-59)$ & $30.0(27-40)$ & 0.003 \\
\hline $\operatorname{AST}(\mathrm{U} / \mathrm{L})(n=177)$ & $57(36-90)$ & $61(36-94)$ & $42(27-62)$ & 0.035 \\
\hline $\operatorname{ALP}(\mathrm{U} / \mathrm{L})(n=187)$ & $124(93-196)$ & $137(95-204)$ & $111(77-150)$ & 0.057 \\
\hline
\end{tabular}

$C D 4=$ cluster of differentiation 4 cells; $W C=$ white cells; $H b=$ haemoglobin; $B U N=$ blood urea nitrogen; $A L T=$ alanine aminotransferase; $A S T=$ aspartate aminotransferase; $A L P=$ alkaline phosphatase.

$I Q R=$ interquartile range.

${ }^{a}$ Mann-Whitney U-test.

(20). Nonetheless, a proportion of patients denied any HIV risk factors, and others presented at an advanced stage and died before verifying any HIV risk factor (reported as "unknown"). This might have led to under-representation of some HIV risk activities previously reported in Libya (21).

Females, on the other hand, were mostly infected with HIV through marital heterosexual transmission, and were more likely to be unaware of their positive HIV disease status before admission. This finding implicates marriage as a potential HIV risk factor, and highlights the importance of pre-marriage counselling and HIV testing for monogamous couples. It also highlights a potential role for public education on the risk of HIV transmission and available protective tools to reduce such risk among serodiscordant couples $(22,23)$. Furthermore, unawareness of HIV status among women in their reproductive years poses a significant risk of mother-to-child-transmission (24), and adds another challenge to national prevention programmes.

In this study, the majority of patients werehospitalized with AIDS-defining illnesses, of which opportunisticinfections were the most common. Pneumocystis jiroveci was the most common respiratory disease, especially among females, while cerebral toxoplasmosis was the most commonly identified intracranial mass lesion. Extrapulmonary TB was more common than pulmonary $\mathrm{TB}$, as previously reported in patients with very low CD4 counts (25). However, findings of such a low prevalence should be interpreted with caution. It is possible that some cases with TB were missed or misclassified as sepsis syndromes, as the only available diagnostic modalities were chest X-ray and sputum for acid-fast bacillus stain; both of which are known to have poor sensitivity in HIV cases, especially at very low CD4 counts $(26,27)$. As reported in previous studies from Africa, implementing new diagnostic modalities such as GeneXpert MTB/RIF (28) and TB-LAM (29) has the potential to diagnose TB among seriously ill patients with severe immunosuppression and may improve their overall prognosis $(30,31)$.

Although the spectrum of opportunistic infections might be comparable across developing countries, the prevalence of the most common opportunistic infections shows wide regional variations. For instance, pulmonary TB was most commonly reported in India
(32,33), Islamic Republic of Iran (14), most countries of Africa $(9,10,17,19)$, Bangladesh (34) and China (13), with very small contributions from Pneumocystis jiroveci $(10,14)$ and toxoplasmosis $(17,35)$. In contrast, the most commonly reported opportunistic infections were Pneumocystis jiroveci in Oman (12), toxoplasmosis in Lebanon (15) and cryptococcal meningitis in Thailand (16).

Knowledge regarding the prevalence of various opportunistic infections among HIV patients in developing countries with limited resources may aid in developing screening protocols, establishing appropriate prevention programmes, and guiding decisions on empirical treatment. It may also allow for better allocation of and prioritization of scarce diagnostic resources.

The risk of and mortality due to sepsis are reported to be higher in HIVpositive compared with HIV-negative patients (36-38). The aetiology of sepsis could also be different, with fungal and mycobacterial infections playing important causative roles $(37,39)$. In our study, the aetiological causes of sepsis could not be ascertained because many inpatients had received various antibiotics before admission, resulting 


\begin{tabular}{|c|c|c|c|c|c|c|c|}
\hline \multirow[t]{2}{*}{ Variable } & \multicolumn{2}{|c|}{ Total } & \multicolumn{2}{|c|}{ Male } & \multicolumn{2}{|c|}{ Female } & \multirow[t]{2}{*}{$P$-value } \\
\hline & No. & $\%$ & No. & $\%$ & No. & $\%$ & \\
\hline CD4 count (cells/ $\mu \mathrm{L})$ & & & & & & & $0.47^{\mathrm{a}}$ \\
\hline$<50$ & 94 & 41.4 & 77 & 41.2 & 17 & 42.5 & \\
\hline $50-200$ & 56 & 24.7 & 48 & 25.7 & 8 & 20.0 & \\
\hline $200-350$ & 11 & 4.8 & 7 & 3.7 & 4 & 10.0 & \\
\hline$>350$ & 8 & 3.5 & 6 & 3.2 & 2 & 5.0 & \\
\hline Missing & 58 & 25.6 & 49 & 26.2 & 9 & 22.5 & \\
\hline WC count $\left(\right.$ cells $\left.\times 10^{3} / \mu L\right)$ & & & & & & & $0.52^{\mathrm{b}}$ \\
\hline$<4$ & 79 & 34.8 & 66 & 35.3 & 13 & 32.5 & \\
\hline $4-11$ & 114 & 50.2 & 93 & 49.7 & 21 & 52.5 & \\
\hline$>11$ & 25 & 11.0 & 22 & 11.8 & 3 & 7.5 & \\
\hline Missing & 9 & 4.0 & 6 & 3.2 & 3 & 7.5 & \\
\hline$H b(g / d L)$ & & & & & & & $0.043^{\mathrm{a}}$ \\
\hline$<8$ & 39 & 17.2 & 36 & 19.3 & 3 & 7.5 & \\
\hline $8-10$ & 68 & 30.0 & 50 & 26.7 & 18 & 45.0 & \\
\hline 10-13 (males);10-12 (females) & 84 & 37.0 & 70 & 37.4 & 14 & 35.0 & \\
\hline > 13 (males); > 12 (males) & 27 & 11.9 & 25 & 13.4 & 2 & 5.0 & \\
\hline Missing & 9 & 4.0 & 6 & 3.2 & 3 & 7.5 & \\
\hline Platelet count $\left(\times 10^{3} / \mu \mathrm{L}\right)$ & & & & & & & $<0.001^{\mathrm{b}}$ \\
\hline$<150$ & 95 & 41.9 & 88 & 47.1 & 7 & 17.5 & \\
\hline $150-400$ & 114 & 50.2 & 90 & 48.1 & 24 & 60.0 & \\
\hline$>400$ & 9 & 4.0 & 3 & 1.6 & 6 & 15.0 & \\
\hline Missing & 9 & 4.0 & 6 & 3.2 & 3 & 7.5 & \\
\hline$B U N(m g / d L)$ & & & & & & & $0.02^{\mathrm{a}}$ \\
\hline$<18$ & 119 & 52.4 & 92 & 49.2 & 27 & 67.5 & \\
\hline$>18$ & 83 & 36.6 & 76 & 40.6 & 7 & 17.5 & \\
\hline Missing & 25 & 11.0 & 19 & 10.2 & 6 & 15.0 & \\
\hline Creatinine $(\mathrm{mg} / \mathrm{dL})$ & & & & & & & $0.15^{\mathrm{a}}$ \\
\hline$<1.3$ & 169 & 74.4 & 137 & 73.3 & 32 & 80.0 & \\
\hline$>1.3$ & 39 & 17.2 & 36 & 19.3 & 3 & 7.5 & \\
\hline Missing & 19 & 8.4 & 14 & 7.5 & 5 & 12.5 & \\
\hline Sodium $(\mathrm{mmol} / \mathrm{L})$ & & & & & & & $0.027^{b}$ \\
\hline$<135$ & 141 & 62.1 & 124 & 66.3 & 17 & 42.5 & \\
\hline 135-145 & 63 & 27.8 & 46 & 24.6 & 17 & 42.5 & \\
\hline$>145$ & 5 & 2.2 & 4 & 2.1 & 1 & 2.5 & \\
\hline Missing & 18 & 7.9 & 13 & 7.0 & 5 & 12.5 & \\
\hline Potassium $(\mathrm{mmol} / \mathrm{L})$ & & & & & & & $0.35^{\mathrm{b}}$ \\
\hline$<3.5$ & 43 & 18.9 & 34 & 18.2 & 9 & 22.5 & \\
\hline $3.5-5.5$ & 158 & 69.6 & 132 & 70.6 & 26 & 65.0 & \\
\hline$>5.5$ & 8 & 3.5 & 8 & 4.3 & 0 & 0.0 & \\
\hline Missing & 18 & 7.9 & 13 & 7.0 & 5 & 12.5 & \\
\hline$A L T(U / L)$ & & & & & & & $0.038^{a}$ \\
\hline$<65$ & 125 & 55.1 & 100 & 53.5 & 25 & 62.5 & \\
\hline$>65$ & 27 & 11.9 & 27 & 14.4 & 0 & 0.0 & \\
\hline Missing & 75 & 33.0 & 60 & 32.1 & 15 & 37.5 & \\
\hline $\operatorname{AST}(U / L)$ & & & & & & & $0.071^{\mathrm{a}}$ \\
\hline$<37$ & 50 & 22 & 41 & 21.9 & 9 & 22.5 & \\
\hline$>37$ & 127 & 55.9 & 110 & 58.8 & 17 & 42.5 & \\
\hline Missing & 50 & 22.0 & 36 & 19.3 & 14 & 35.0 & \\
\hline$A L P(U / L)$ & & & & & & & $0.029^{\mathrm{a}}$ \\
\hline$<136$ & 100 & 44.1 & 78 & 41.7 & 22 & 55.0 & \\
\hline$>136$ & 87 & 38.3 & 79 & 42.2 & 8 & 20.0 & \\
\hline Missing & 40 & 17.6 & 30 & 16.0 & 10 & 25.0 & \\
\hline
\end{tabular}

${ }^{a}$ Chi-squared test; ${ }^{b}$ Fisher exact test;

$C D 4=$ cluster of differentiation 4 cells; $W C=$ white cells; $H b=$ haemoglobin; $B U N=$ blood urea nitrogen; $A L T=$ alanine aminotransferase $; A S T=$ aspartate aminotransferase; $A L P=$ alkaline phosphatase. 


\begin{tabular}{|c|c|c|c|c|}
\hline Variable & Crude OR $(95 \% \mathrm{Cl})$ & $P$-value & Adjusted OR (95\% Cl) & $P$-value \\
\hline \multicolumn{5}{|l|}{ HAART use } \\
\hline Never & ref. & & & \\
\hline Current & $0.44(0.22-0.91)$ & 0.023 & $0.33(0.14-0.80)$ & 0.015 \\
\hline Defaulted & $1.02(0.45-2.35)$ & 0.96 & $1.28(0.49-3.40)$ & 0.62 \\
\hline Unknown & $1.42(0.09-23.4)$ & 0.81 & $2.11(0.08-53.9)$ & 0.65 \\
\hline Central nervous system symptoms ${ }^{a}$ & $2.59(1.36-4.95)$ & 0.005 & $4.12(1.77-9.60)$ & 0.001 \\
\hline Sepsis & $11.5(3.19-41.5)$ & $<0.001$ & $6.98(1.71-28.4)$ & 0.007 \\
\hline \multicolumn{5}{|l|}{ Total lymphocyte count $\left(\text { cells } \times 10^{3} / \mu L\right)^{b}$} \\
\hline $1-3$ & ref. & & & \\
\hline$<1$ & $2.74(1.26-5.98)$ & 0.011 & $3.60(1.36-9.52)$ & 0.01 \\
\hline$>3$ & $1.95(0.41-9.19)$ & 0.4 & $1.71(0.27-10.9)$ & 0.57 \\
\hline \multicolumn{5}{|l|}{$B U N(m g / d L)^{a}$} \\
\hline$<18$ & ref. & & & \\
\hline$>18$ & $2.36(1.27-4.39)$ & 0.008 & $1.58(0.67-3.77)$ & 0.3 \\
\hline \multicolumn{5}{|l|}{ Creatinine $(\mathrm{mg} / \mathrm{dL})^{b}$} \\
\hline$<1.3$ & ref. & & & \\
\hline$>1.3$ & $3.05(1.43-6.50)$ & 0.003 & $1.88(0.64-5.49)$ & 0.25 \\
\hline \multicolumn{5}{|l|}{ Sodium $(\mathrm{mmol} / \mathrm{L})^{b}$} \\
\hline 135-145 & ref. & & & \\
\hline$<135$ & $2.01(1.00-4.07)$ & 0.056 & $2.69(1.12-6.44)$ & 0.027 \\
\hline$>145$ & $2.00(0.30-13.2)$ & 0.47 & $0.45(0.05-4.10)$ & 0.48 \\
\hline \multicolumn{5}{|l|}{ Potassium $(\mathrm{mmol} / \mathrm{L})^{b}$} \\
\hline $3.5-5.5$ & ref. & & & \\
\hline$<3.5$ & $2.57(1.23-5.34)$ & 0.011 & $2.71(1.14-6.41)$ & 0.023 \\
\hline$>5.5$ & $2.31(0.45-11.9)$ & 0.31 & $1.93(0.33-11.2)$ & 0.46 \\
\hline
\end{tabular}

${ }^{a}$ Central nervous system symptoms included: coma, convulsions or focal neurological deficits; ${ }^{b}$ Laboratory normal ranges for each test were used as reference groups. $H A A R T=$ highly active antiretroviral therapy; $B U N=$ blood urea nitrogen; ref. = reference group. $O R=$ odds ratio; $C I=$ confidence interval.

in negative blood culture results. Also special diagnostic techniques for TB, fungi and other atypical organisms known to cause sepsis among HIV patients were not available due to resource constraints. Consequently, our results might not be sufficient to clearly describe the spectrum of TB, atypical organisms and disseminated fungal infections among HIV inpatients.

Despite several years availability of HAART in Libya, late presentation and poor HAART uptake by patients were common; a finding similar to previous reports (40-42). Late presentation is known to be associated with poor response to HAART (43), increased mortality (especially shortly after commencing HAART) (11,40-42,44-46), and high cumulative risk of HIV transmission (47).

Furthermore, the finding that the majority of patients were known to be HIV-positive and yet were not on HAART highlights another significant gap in access to and retention of HIV care services. Previous studies have identified several factors as potentially contributing to late presentation, poor HAART uptake and low retention in HIV care. These factors include stigma, fear of status disclosure, complexity of treatment regimens and lack of psychosocial support (48); lack of confidentiality and fear of discrimination at health facilities, workplace and community (49); poor staff-patient relationships and underestimation of the need for HAART (50); and active drug use $(43,46)$.

Stigma and fear of disclosure are particularly important among females living in a conservative society. Previous studies from other Arab countries have reported a high prevalence of stigma against HIV-positive patients and poor knowledge about the disease, especially among females (51-53).

Health system strategies and HIV service delivery interventions such as counselling, brief case management 
discussion with patients, simplified treatment regimens, screening and management of depression, and co-location with drug rehabilitation services could potentially improve access to and retention in HIV care, reduce late presentation and increase HAART uptake $(54,55)$.

The fact that some patients were not aware of their HIV disease status until the development of opportunistic infections might also reflect low education and lack of awareness about the risk of HIV transmission. This could potentially be ameliorated through education programmes and HIV awareness campaigns targeting the general population, accompanied by screening high-risk groups such as injection drug users and sexually active youth. Once diagnosed, patients should then be promptly referred to specialist HIV care.

Anaemia was identified in the majority of our inpatients and was severe enough in $17 \%$ of them to require blood transfusion. Anaemia has previously been reported in many developed and developing countries $(13,19,56)$ and has been linked with HIV-disease progression and poor outcomes (57-59). In the context of advanced medical illnesses such as AIDS, anaemia could be due to poor nutrition, infections such as candidiasis and $\mathrm{TB}$, or the advanced stage of HIV disease itself $(60,61)$.

We reported a high rate of in-hospital mortality of $37.4 \%$, which was broadly similar to other developing countries where AIDS is still the main cause for hospitalization $(9,17,19,32,62)$. By contrast, in developed countries during the era of HAART, in-hospital mortality is very low and it is mostly related to non-AIDS illnesses such as malignancy and cardiovascular diseases $(4,6,7,63)$.

In this study, HAART use was associated with a reduced risk of mortality, a finding consistent with previous reports on improved survival among
HIV patients receiving HAART (3,64-66). In addition, a presentation with central nervous system symptoms, development of hyponatraemia or a diagnosis of sepsis were independently associated with increased risk of mortality, generally reflecting the severity of underlying diseases $(67,68)$. The lack of appropriate diagnostic tools (brain biopsy, GeneXpert MTB/ RIF, TB LAM, special culture media for atypical pathogens) could have led to a delay in establishing diagnoses and late initiation of aetiology-specific treatments, which could have negatively affected patients' overall prognosis. In addition, a low total lymphocyte count, which serves as a valid surrogate indicator of immunosuppression in resource-limited settings (69-71), was also associated with increased mortality, emphasizing the impact of severe immunosuppression on HIV-related mortality $(4,7,9,19)$. Patient's sex, $\mathrm{HCV}$ coinfection and anaemia were not predictive of mortality in this study. This could be due to the small number of females included, and the high prevalence of both HCV and anaemia among the study participants.

Our study has some limitations that should be acknowledged. The study was conducted retrospectively and some data on clinical and laboratory variables were missing. This study was of patients admitted to a tertiary health-care centre and its results may not be generalizable, as such settings tend to receive seriously ill cases who need specialized care and management. In some instances, a definitive diagnosis could not be reached. This might have been due to the serious and advanced condition of patients at presentation, which prevented further invasive diagnostics, or due to inadequate diagnostic facilities (e.g. culture media for atypical organisms); unavailability of diagnostic facilities (e.g. brain biopsy and histopathological diagnosis of cerebral toxoplasmosis, central nervous system TB and progressive multifocal leukoencephalopathy) and other resource constrains. Additionally, cause of death could not be confirmed due to lack of permissions for post-mortem studies, raising a possibility that other diagnoses (which might have been missed ante-mortem) might have also influenced patient outcomes (72-74).

Despite the limitations, our study has important strengths. To our knowledge, this is the first study to describe clinical, immunological and biochemical profiles and examine factors associated with mortality among hospitalized adults with HIV/AIDS in Libya. Our study also had a high-level representation on HIV-related hospital admissions, as Tripoli Medical Centre is one of the only 2 centres offering HIV care in western Libya.

\section{Conclusion}

The issue of late presentation and AIDS-related mortality is challenging and requires coordinated approaches and collaboration among various stakeholders. More efforts should be placed on improving access to HIV care, especially for females and marginalized risk groups such as injection drug use and homosexual men, successful linkage to care after diagnosis, prompt initiation of HAART, and retention on treatment and in care. There should also be strategies to integrate HIV treatment centres with mental health and drug rehabilitation services. Additionally, increasing HIV awareness and education in the community, screening high-risk populations, and improving the diagnostic capacity of referral laboratories might help in changing the current situation of HIV wards in Libya.

\section{Acknowledgements}

Funding: None.

Competing interests: None declared. 
1. Global report: UNAIDS report on the global AIDS epidemic 2013. Geneva Joint United Nations Programme on HIV/AIDS; 2013.

2. Bonnet F, Burty C, Lewden C, Costagliola D, May T, Bouteloup $V$, et al.; Agence Nationale de Recherches sur le Sida et les Hépatites Virales EN19 Mortalité Study Group; Mortavic Study Group. Changes in cancer mortality among HIV-infected patients: the Mortalité 2005 Survey. Clin Infect Dis. 2009 Mar 1;48(5):633-9. PMID:19202627

3. Krentz HB, Kliewer G, Gill MJ. Changing mortality rates and causes of death for HIV-infected individuals living in Southern Alberta, Canada from 1984 to 2003. HIV Med. 2005 Mar;6(2):99-106. PMID:15807715

4. Kim JH, Psevdos G Jr, Gonzalez E, Singh S, Kilayko MC, Sharp V. All-cause mortality in hospitalized HIV-infected patients at an acute tertiary care hospital with a comprehensive outpatient HIV care program in New York City in the era of highly active antiretroviral therapy (HAART). Infection. 2013 Apr;41(2):54551. PMID:23264096

5. Sackoff JE, Hanna DB, Pfeiffer MR, Torian LV. Causes of death among persons with AIDS in the era of highly active antiretroviral therapy: New York City. Ann Intern Med. 2006 Sep 19;145(6):397-406. PMID:16983127

6. Rapp C, Reggad A, Aoun A, Ficko C, Andriamanantena D, Flateau C. Hospitalization causes of HIV-infected patients in 2011 in an HIV reference center in the Paris region, France. J Int AIDS Soc. 2012;15(Suppl 4):18126.

7. Falster K, Wand H, Donovan B, Anderson J, Nolan D, Watson $\mathrm{K}$, et al.; Australian HIV Observational Database. Hospitalizations in a cohort of HIV patients in Australia, 1999-2007. AIDS. 2010 Jun 1;24(9):1329-39. PMID:20559038

8. Buchacz K, Baker RK, Moorman AC, Richardson JT, Wood KC, Holmberg SD, et al.; HIV Outpatient Study (HOPS) Investigators. Rates of hospitalizations and associated diagnoses in a large multisite cohort of HIV patients in the United States, 1994-2005. AIDS. 2008 Jul 11;22(11):1345-54. PMID:18580614

9. Agaba PA, Digin E, Makai R, Apena L, Agbaji OO, Idoko JA et al. Clinical characteristics and predictors of mortality in hospitalized HIV-infected Nigerians. J Infect Dev Ctries. 2011 May;5(5):377-82. PMID:21628815

10. Colvin M, Dawood S, Kleinschmidt I, Mullick S, Lallo U. Prevalence of HIV and HIV-related diseases in the adult medical wards of a tertiary hospital in Durban, South Africa. Int J STD AIDS. 2001 Jun;12(6):386-9. PMID:11368820

11. Namutebi AMN, Kamya MRK, Byakika-Kibwika P. Causes and outcome of hospitalization among HIV-infected adults receiving antiretroviral therapy in Mulago hospital, Uganda. Afr Health Sci. 2013 Dec;13(4):977-85. PMID:24940321

12. Balkhair AA, Al-Muharrmi ZK, Ganguly S, Al-Jabri AA. Spectrum of AIDS Defining Opportunistic Infections in a Series of 77 Hospitalised HIV-infected Omani Patients. Sultan Qaboos Univ Med J. 2012 Nov;12(4):442-8. PMID:23275840

13. Xiao J, Gao G, Li Y, Zhang W, Tian Y, Huang Y, et al. Spectrums of opportunistic infections and malignancies in HIVinfected patients in tertiary care hospital, China. PLoS One. 2013;8(10):e75915. PMID:24204583

14. Sharifi-Mood B, Alavi-Naini R, Salehi M, Hashemi M, Rakhshani F. Spectrum of clinical disease in a series of hospitalized HIV-infected patients from southeast of Iran. Saudi Med J. 2006 Sep;27(9):1362-6. PMID:16951774

15. Naba MR, Kanafani ZA, Awar GN, Kanj SS. Profile of opportunistic infections in HIV-infected patients at a tertiary care center in Lebanon. J Infect Public Health. 2010;3(3):130-3. PMID:20869674
16. Inverarity $\mathrm{D}$, Bradshaw $\mathrm{Q}$, Wright $\mathrm{P}$, Grant $\mathrm{A}$. The spectrum of HIV-related disease in rural Central Thailand. Southeast Asian J Trop Med Public Health. 2002 Dec;33(4):822-31. PMID:12757233

17. Thinyane K, Cooper V. Clinical profiles of HIV-infected, HAART-naive patients admitted to a tertiary level hospital in Maseru, Lesotho. Int J Infect Dis. 2013;11(1):1.

18. UNAIDS 2013 regional report for the Middle East and North Africa. Cairo, Egypt: Joint United Nations Programme on HIV/ AIDS; 2013.

19. Ogoina D, Obiako RO, Muktar HM, Adeiza M, Babadoko A, Hassan A, et al. Morbidity and Mortality Patterns of Hospitalized Adult HIV/AIDS Patients in the Era of Highly Active Antiretroviral Therapy: A 4-year Retrospective Review from Zaria, Northern Nigeria. AIDS Res Treat. 2012;2012.

20. Mirzoyan L, Berendes S, Jeffery C, Thomson J, Ben Othman H, Danon L, et al. New evidence on the HIV epidemic in Libya: why countries must implement prevention programs among people who inject drugs. J Acquir Immune Defic Syndr. 2013 Apr 15;62(5):577-83. PMID:23337363

21. Valadez JJ, Berendes S, Jeffery C, Thomson J, Ben Othman H, Danon L, et al. Filling the Knowledge Gap: Measuring HIV Prevalence and Risk Factors among Men Who Have Sex with Men and Female Sex Workers in Tripoli, Libya. PLoS One. 2013;8(6):e66701. PMID:23840521

22. Curran K, Baeten JM, Coates TJ, Kurth A, Mugo NR, Celum C. HIV-1 prevention for HIV-1 serodiscordant couples. Curr HIV/ AIDS Rep. 2012 Jun;9(2):160-70. PMID:22415473

23. Allen S, Meinzen-Derr J, Kautzman M, Zulu I, Trask S, Fideli U, et al. Sexual behavior of HIV discordant couples after HIV counseling and testing. AIDS. 2003 Mar 28;17(5):733-40. PMID:12646797

24. da Cruz Gouveia PA, da Silva GAP, de Fatima Pessoa Militão de Albuquerque M. Factors associated with mother-to-child transmission of the human immunodeficiency virus in Pernambuco, Brazil, 2000-2009. Trop Med Int Health. 2013 Mar;18(3):276-85. PMID:23279690

25. Naing C, Mak JW, Maung M, Wong SF, Kassim AIBM. Metaanalysis: the association between HIV infection and extrapulmonary tuberculosis. Lung. 2013 Feb;191(1):27-34. PMID:23180033

26. Chamie G, Luetkemeyer A, Walusimbi-Nanteza M, Okwera A, Whalen CC, Mugerwa RD, et al. Significant variation in presentation of pulmonary tuberculosis across a high resolution of CD4 strata. Int J Tuberc Lung Dis. 2010 Oct;14(10):1295-302. PMID:20843421

27. Mugusi F, Villamor E, Urassa W, Saathoff E, Bosch RJ, Fawzi WW. HIV co-infection, CD4 cell counts and clinical correlates of bacillary density in pulmonary tuberculosis. Int J Tuberc Lung Dis. 2006 Jun;10(6):663-9. PMID:16776454

28. Scott LE, McCarthy K, Gous N, Nduna M, Van Rie A, Sanne I, et al. Comparison of Xpert MTB/RIF with other nucleic acid technologies for diagnosing pulmonary tuberculosis in a high HIV prevalence setting: a prospective study. PLoS Med. 2011 Jul;8(7):e1001061. PMID:21814495

29. Lawn SD, Kerkhoff AD, Vogt M, Wood R. Diagnostic accuracy of a low-cost, urine antigen, point-of-care screening assay for HIV-associated pulmonary tuberculosis before antiretroviral therapy: a descriptive study. Lancet Infect Dis. 2012 Mar;12(3):201-9. PMID:22015305

30. Lawn SD, Kerkhoff AD, Vogt M, Wood R. HIV-associated tuberculosis: relationship between disease severity and the sensitivity of new sputum-based and urine-based diagnostic assays. BMC Med. 2013;11:231. PMID:24168211 
31. Peter JG, Theron G, Dheda K. Can point-of-care urine LAM strip testing for tuberculosis add value to clinical decision making in hospitalised HIV-infected persons? PLoS One. 2013;8(2):e54875. PMID:23390504

32. Sobhani R, Basavaraj A, Gupta A, Bhave AS, Kadam DB, Sangle SA, et al. Mortality and clinical characteristics of hospitalized adult patients with HIV in Pune, India. Indian J Med Res. 2007 Aug;126(2):116-21. PMID:17932435

33. Sharma SK, Kadhiravan T, Banga A, Goyal T, Bhatia I, Saha PK. Spectrum of clinical disease in a series of 135 hospitalised HIVinfected patients from north India. BMC Infect Dis. 2004 Nov 22;4:52. PMID:15555069

34. Matin N, Shahrin L, Pervez MM, Banu S, Ahmed D, Khatun M, et al. Clinical profile of HIV/AIDS-infected patients admitted to a new specialist unit in Dhaka, Bangladesh-a low-prevalence country for HIV. J Health Popul Nutr. 2011 Feb;29(1):14-9 PMID:21528786

35. Liwlompaisan W, Kiertiburanakul S. Causes of hospitalizations among HIV-infected adults in the highly active antiretroviral therapy era in Thailand. J Infect Dis Antimicrob Agents. 2013;30:5-14.

36. Mrus JM, Braun L, Yi MS, Linde-Zwirble WT, Johnston JA. Impact of HIV/AIDS on care and outcomes of severe sepsis. Crit Care. 2005;9(6):R623-30. PMID:16280060

37. Silva JM Jr, dos Santos SdeS. Sepsis in AIDS patients: clinical, etiological and inflammatory characteristics. J Int AIDS Soc. 2013;16:17344. PMID:23374857

38. Alberti C, Brun-Buisson C, Burchardi H, Martin C, Goodman S Artigas A, et al. Epidemiology of sepsis and infection in ICU patients from an international multicentre cohort study. Intensive Care Med. 2002 Feb;28(2):108-21. PMID:11907653

39. Crump JA, Ramadhani HO, Morrissey AB, Saganda W, Mwako MS, Yang L-Y, et al. Invasive bacterial and fungal infections among hospitalized HIV-infected and HIV-uninfected adults and adolescents in northern Tanzania. Clin Infect Dis. 2011 Feb 1;52(3):341-8. PMID:21217181

40. Mocroft A, Lundgren JD, Sabin ML, Monforte Ad, Brockmeyer $\mathrm{N}$, Casabona J, et al.; Collaboration of Observational HIV Epidemiological Research Europe (COHERE) study in EuroCoord. Risk factors and outcomes for late presentation for HIV-positive persons in Europe: results from the Collaboration of Observational HIV Epidemiological Research Europe Study (COHERE). PLoS Med. 2013;10(9):e1001510. PMID:24137103

41. d'Arminio Monforte A, Cozzi-Lepri A, Girardi E, Castagna A, Mussini C, Di Giambenedetto S, et al.; Icona Foundation Study Group. Late presenters in new HIV diagnoses from an Italian cohort of HIV-infected patients: prevalence and clinical outcome. Antivir Ther. 2011;16(7):1103-12. PMID:22024526

42. Camoni L, Raimondo M, Regine V, Salfa MC, Suligoi B; regional representatives of the HIV Surveillance System. Late presenters among persons with a new HIV diagnosis in Italy, 2010-2011. BMC Public Health. 2013;13:281. PMID:23537210

43. Rodríguez-Arenas MA, Jarrín I, del Amo J, Iribarren JA, Moreno S, Viciana P, et al.; CoRIS-MD. Delay in the initiation of HAART, poorer virological response, and higher mortality among HIVinfected injecting drug users in Spain. AIDS Res Hum Retroviruses. 2006 Aug;22(8):715-23. PMID:16910826

44. Lawn SD, Harries AD, Anglaret X, Myer L, Wood R. Early mortality among adults accessing antiretroviral treatment programmes in sub-Saharan Africa. AIDS. 2008 Oct 1;22(15):1897908. PMID:18784453

45. Egger M, May M, Chêne G, Phillips AN, Ledergerber B, Dabis F, et al.; ART Cohort Collaboration. Prognosis of HIV-1-infected patients starting highly active antiretroviral therapy: a collaborative analysis of prospective studies. Lancet. 2002 Jul 13;360(9327):119-29. PMID:12126821
46. Sobrino-Vegas P, García-San Miguel L, Caro-Murillo AM, Miró JM, Viciana P, Tural C, et al.; CoRIS. Delayed diagnosis of HIV infection in a multicenter cohort: prevalence, risk factors, response to HAART and impact on mortality. Curr HIV Res. 2009 Mar;7(2):224-30. PMID:19275591

47. Girardi E, Sabin CA, Monforte AD. Late diagnosis of HIV infection: epidemiological features, consequences and strategies to encourage earlier testing. J Acquir Immune Defic Syndr. 2007 Sep;46 Suppl 1:S3-8. PMID:17713423

48. Mills EJ, Nachega JB, Bangsberg DR, Singh S, Rachlis B, Wu P, et al. Adherence to HAART: a systematic review of developed and developing nation patient-reported barriers and facilitators. PLoS Med. 2006 Nov;3(11):e438. PMID:17121449

49. Retention in HIV programmes: defining the challenges and identifying solutions. Meeting report, 13-15 September 2011. Geneva: World Health Organization; 2012.

50. Langebeek N, Gisolf EH, Reiss P, Vervoort SC, Hafsteinsdóttir TB, Richter C, et al. Predictors and correlates of adherence to combination antiretroviral therapy (ART) for chronic HIV infection: a meta-analysis. BMC Med. 2014;12:142. PMID:25145556

51. Gańczak M, Barss P, Alfaresi F, Almazrouei S, Muraddad A, AlMaskari F. Break the silence: HIV/AIDS knowledge, attitudes, and educational needs among Arab university students in United Arab Emirates. J Adolesc Health. 2007 Jun;40(6):572. e1-8. PMID:17531765

52. Badahdah AM, Sayem N. HIV-related knowledge and AIDS stigma among college students in Yemen. East Mediterr Health J. 2010 Aug;16(8):901-6. PMID:21469573

53. Badahdah AM, Foote CE. Role of shame in the stigmatization of people with human immunodeficiency virus: a survey of female college students in 3 Arab countries. East Mediterr Health J. 2010 Sep;16(9):982-7. PMID:21218727

54. Thompson MA, Mugavero MJ, Amico KR, Cargill VA, Chang LW, Gross R, et al. Guidelines for improving entry into and retention in care and antiretroviral adherence for persons with HIV: evidence-based recommendations from an International Association of Physicians in AIDS Care panel. Ann Intern Med. 2012 Jun 5;156(11):817-33, W-284, W-285, W-286, W-287, W-288, W-289, W-290, W-291, W-292, W-293, W-294. PMID:22393036

55. Brennan A, Browne JP, Horgan M. A systematic review of health service interventions to improve linkage with or retention in HIV care. AIDS Care. 2014;26(7):804-12. PMID:24354712

56. Sullivan PS, Hanson DL, Chu SY, Jones JL, Ward JW. Epidemiology of anemia in human immunodeficiency virus (HIV)-infected persons: results from the multistate adult and adolescent spectrum of HIV disease surveillance project. Blood. 1998 Jan 1;91(1):301-8. PMID:9414298

57. Harris RJ, Sterne JAC, Abgrall S, Dabis F, Reiss P, Saag M, et al.; Antiretroviral Therapy Cohort Collaboration. Prognostic importance of anaemia in HIV type-1-infected patients starting antiretroviral therapy: collaborative analysis of prospective cohort studies. Antivir Ther. 2008;13(8):959-67. PMID:19195321

58. Mocroft A, Lifson AR, Touloumi G, Neuhaus J, Fox Z, Palfreeman A, et al.; INSIGHT SMART Study Group. Haemoglobin and anaemia in the SMART study. Antivir Ther. 2011;16(3):329-37. PMID:21555815

59. Mocroft A, Kirk O, Barton SE, Dietrich M, Proenca R, Colebunders $\mathrm{R}$, et al. Anaemia is an independent predictive marker for clinical prognosis in HIV-infected patients from across Europe. EuroSIDA study group. AIDS. 1999 May 28;13(8):943-50. PMID:10371175

60. Redig AJ, Berliner N. Pathogenesis and clinical implications of HIV-related anemia in 2013. Hematology Am Soc Hematol Educ Program. 2013;2013:377-81. PMID:24319207 
61. Rawat R, McCoy SI, Kadiyala S. Poor diet quality is associated with low CD4 count and anemia and predicts mortality among antiretroviral therapy-naive HIV-positive adults in Uganda. J Acquir Immune Defic Syndr. 2013 Feb 1;62(2):24653. PMID:23117502

62. Saleri N, Capone S, Pietra V, De laco G, Del Punta V, Rizzi M, et al. Outcome and predictive factors of mortality in hospitalized HIV-patients in Burkina Faso. Infection. 2009 Apr;37(2):142-7. PMID:19169635

63. Smith CJ, Ryom L, Weber R, Morlat P, Pradier C, Reiss P, et al.; D:A:D Study Group. Trends in underlying causes of death in people with HIV from 1999 to 2011 (D:A:D): a multicohort collaboration. Lancet. 2014 Jul 19;384(9939):241-8. PMID:25042234

64. Chkhartishvili N, Sharvadze L, Chokoshvili O, Bolokadze N, Rukhadze N, Kempker RR, et al. Mortality and causes of death among HIV-infected individuals in the country of Georgia: 1989-2012. AIDS Res Hum Retroviruses. 2014 Jun;30(6):560-6. PMID:24472093

65. Weber R, Ruppik M, Rickenbach M, Spoerri A, Furrer H, Battegay M, et al.; Swiss HIV Cohort Study (SHCS). Decreasing mortality and changing patterns of causes of death in the Swiss HIV Cohort Study. HIV Med. 2013 Apr;14(4):195-207. PMID:22998068

66. Yang $\mathrm{CH}$, Huang YF, Hsiao CF, Yeh YL, Liou HR, Hung CC, et al. Trends of mortality and causes of death among HIV-infected patients in Taiwan, 1984-2005. HIV Med. 2008 Aug;9(7):53543. PMID:18554309

67. Dao CN, Peters PJ, Kiarie JN, Zulu I, Muiruri P, Ong'ech J, et al. Hyponatremia, hypochloremia, and hypoalbuminemia predict an increased risk of mortality during the first year of antiretroviral therapy among HIV-infected Zambian and Kenyan women. AIDS Res Hum Retroviruses. 2011 Nov;27(11):1149-55. PMID:21417949

68. Japiassú AM, Amâncio RT, Mesquita EC, Medeiros DM, Bernal $\mathrm{HB}$, Nunes EP, et al. Sepsis is a major determinant of outcome in critically ill HIV/AIDS patients. Crit Care. 2010;14(4):R152. PMID:20698966

69. Obirikorang C, Quaye L, Acheampong I. Total lymphocyte count as a surrogate marker for CD4 count in resource-limited settings. BMC Infect Dis. 2012;12:128. PMID:22676809

70. Prajapati S. Utility of total lymphocyte count as an affordable surrogate for CD4 lymphocyte count in HIV infected Nepali patients. J AIDS Clin Res. 2014;05(03).

71. Mahajan AP, Hogan JW, Snyder B, Kumarasamy N, Mehta $\mathrm{K}$, Solomon $\mathrm{S}$, et al. Changes in total lymphocyte count as a surrogate for changes in CD4 count following initiation of HAART: implications for monitoring in resource-limited settings. J Acquir Immune Defic Syndr. 2004 May 1;36(1):567-75. PMID:15097299

72. Tang H-J, Liu Y-C, Yen M-Y, Chen Y-S, Wann S-R, Lin H-H, et al. Opportunistic infections in adults with acquired immunodeficiency syndrome: a comparison of clinical and autopsy findings. J Microbiol Immunol Infect. 2006 Aug;39(4):310-5. PMID:16926977

73. Cox JA, Lukande RL, Lucas S, Nelson AM, Van Marck E, Colebunders R. Autopsy causes of death in HIV-positive individuals in sub-Saharan Africa and correlation with clinical diagnoses. AIDS Rev. 2010 Oct-Dec;12(4):183-94. PMID:21179183

74. Cox JA, Lukande RL, Nelson AM, Mayanja-Kizza H, Colebunders R, Van Marck E, et al. An autopsy study describing causes of death and comparing clinico-pathological findings among hospitalized patients in Kampala, Uganda. PLoS One. 2012;7(3):e33685. PMID:22432042 